Pacific

Journal of

Mathematics

\title{
DIRICHLET FORMS ON THE SIERPIŃSKI GASKET
}

Robert Meyers, Robert S. Strichartz and Alexander Teplyaev 


\title{
DIRICHLET FORMS ON THE SIERPIŃSKI GASKET
}

\author{
Robert Meyers, Robert S. Strichartz and Alexander Teplyaev
}

\begin{abstract}
We study not necessarily self-similar Dirichlet forms on the Sierpiński gasket that can be described as limits of compatible resistance networks on the sequence of graphs approximating the gasket. We describe the compatibility conditions in detail, and we also present an alternative description, based on just 3 conductance values and the 3-dimensional space of harmonic functions. In addition, we show how to parameterize all the Dirichlet forms by a set of independent variables.
\end{abstract}

\section{Introduction}

The central object in the theory of analysis on fractals, as it has developed so far, is the Dirichlet form, analogous to $\int|\nabla f|^{2} d x$ in the theory of Riemannian manifolds. Great effort has gone into the construction of self-similar Dirichlet forms on self-similar fractals, with the successful theory of Kigami [Ki2] covering a class of finitely ramified fractals called post-critically finite (pcf). This class of fractals includes the familiar Sierpiński gasket (SG), and Kigami's approach is based on his original construction for SG in [Ki1], which was completed by Sabot [Sa] with the classification of all self-similar Dirichlet forms on SG (see also [Me, SST, Te]). These Dirichlet forms have the property that points have positive capacity. In general this property is true for regular harmonic structures and is not true for nonregular harmonic structures. We will discuss this in more detail in Section 7.

The goal of this paper is to describe the class of all Dirichlet forms on SG - we drop the self-similarity requirement on the form, though of course $\mathrm{SG}$ retains its topological self-similarity. It should be understood that the geometric structure of SG inherited from the standard realization in the plane plays no role in this theory. Instead, in Section 6 we use the harmonic coordinates realization of SG from [Ki3]. However, the question of what is the "correct" geometry for SG, or what might be the analog of a Riemannian metric, is not resolved at present. Following our motivational analogy a step further, we might imagine that the class of pcf self-similar fractals plays the role of symmetric spaces in Riemannian geometry, and SG plays the role of the sphere. The self-similarity condition on the Dirichlet form might be analogous to group invariance of the Riemannian metric on a symmetric space. This suggests that an understanding of the special case of SG might 
yield useful insights into the problem of describing all Dirichlet forms on the general finitely ramified fractal (again there is no generally accepted definition of this class of fractals).

In a crude sense, there already exists a solution to our problem. Consider the sequence of graphs $\left\{\Gamma_{n}\right\}$, where $\Gamma_{0}$ is the complete graph on the vertices $V_{0}=\left\{q_{1}, q_{2}, q_{3}\right\}$, the boundary points of the triangle containing $\mathrm{SG}$, and $\Gamma_{n}$ is defined inductively to contain the vertices

$$
V_{n}=\bigcup_{i=1}^{3} F_{i} V_{n-1},
$$

where $F_{i} x=\frac{1}{2}\left(x+q_{i}\right)$ and the edge relation $x \sim_{j} y$ holds if there exists $F_{i}$ and $x^{\prime}, y^{\prime} \in V_{n-1}$ with $x^{\prime} \sim_{j-1} y^{\prime}$ and $x=F_{i} x^{\prime}, y=F_{i} y^{\prime}$. The sets of vertices $V_{n}$ are contained in SG, and SG is the closure of $V_{*}=\bigcup V_{n}$ in a suitable topology. We will say that a Dirichlet form is point-sensitive if it assigns positive capacity to all points in $V_{*}$. Given any Dirichlet form $\mathcal{E}$ on $\mathrm{SG}$ with domain dom $\mathcal{E}$, we can define its restriction to $\Gamma_{n}$ as follows: for any function $f$ on $V_{n}$ define its $n$-harmonic extension $\widetilde{f}$ to SG to be the element in $\operatorname{dom} \mathcal{E}$ satisfying $\left.\widetilde{f}\right|_{V_{n}^{\prime}}=f$ and minimizing $\mathcal{E}(\widetilde{f}, \widetilde{f})$. Note that it is important that the Dirichlet form is point-sensitive for this definition to make sense. (For example, in a planar region for the usual Dirichlet form, no such minimizer exists.) The restriction $\mathcal{E}_{n}$ is defined by $\mathcal{E}_{n}(f, f)=\mathcal{E}(\widetilde{f}, \widetilde{f})$, and similarly for the bilinear form $\mathcal{E}_{n}(f, g)=\mathcal{E}(\widetilde{f}, \widetilde{g})$. Then $\mathcal{E}_{n}$ is a Dirichlet form on $\Gamma_{n}$ and so can be written

$$
\mathcal{E}_{n}(f, f)=\sum_{x \sim_{n} y} c(x, y)(f(x)-f(y))^{2}
$$

for certain positive conductance coefficients $c(x, y)$. The reciprocal of a conductance is a resistance, and the graph $\Gamma_{n}$ equipped with the Dirichlet form (1.1) is called a resistance network. In this paper we study pointsensitive Dirichlet forms in terms the approximating sequence of Dirichlet forms $\mathcal{E}_{n}$.

The Dirichlet forms $\mathcal{E}_{n}$ satisfy a compatibility condition: the restriction of $\mathcal{E}_{n}$ to $\Gamma_{n-1}$ must be equal to $\mathcal{E}_{n-1}$. This may be expressed as an algebraic condition relating the conductances on $V_{n}$ and $V_{n-1}$. In fact it suffices to understand this condition on the first level, $n=1$, since it is a local condition on each cell $F_{w} V_{0}$, where $F_{w}=F_{w_{1}} \cdots F_{w_{n}}$ for any word $w_{1} \cdots w_{n}$ of length $|w|=n$ from the alphabet $\{1,2,3\}$. For $n=1$ this becomes a polynomial equation in the 9 conductances on $V_{1}$ in terms of the 3 conductances on $V_{0}$. We will show that for each choice of $V_{0}$ conductances the solution set is a 6-dimensional manifold by giving an explicit parameterization in Section 5 . We give a geometric interpretation in Section 6. Nevertheless, the algebraic structure of the compatibility conditions is still somewhat mysterious. 
A new description of Dirichlet forms that we present involves the harmonic functions. These are just the harmonic extensions from $\Gamma_{0}$, and so form a 3 -dimensional space $\mathcal{H}$, each $h$ in $\mathcal{H}$ being determined by its values on $V_{0}$, which is regarded as the boundary of SG. (In general, harmonic extensions from $\Gamma_{n}$ give functions that are harmonic everywhere except at the points of $V_{n}$.) Of course $\mathcal{H}$ contains the constants, since these have zero energy. A basis for $\mathcal{H}$ consists of the functions $h_{k}$ such that $h_{k}\left(q_{j}\right)=\delta_{k j}$, and $h_{1}+h_{2}+h_{3}=1$.

One easy consequence of this description is that $\mathcal{E}$ is determined by the space $\mathcal{H}$ and the 3 conductance coefficients $d_{1}=c\left(q_{2}, q_{3}\right), d_{2}=c\left(q_{1}, q_{3}\right)$, $d_{3}=c\left(q_{1}, q_{2}\right)$. We will demonstrate this in a constructive manner by deriving an algebraic formula for the 9 conductances on $V_{1}$ in terms of this data (more precisely, the coefficients $d_{k}$ and the values of $h_{k}$ at the points in $V_{1}$ ). The same formula may be used inductively to find all coefficients of the restrictions $\mathcal{E}_{n}$, using the values of $h_{k}$ on points in $V_{n}$.

To complete the description it would be desirable to have necessary and sufficient conditions on the data to be associated with a Dirichlet form, but we have been unable to do this. We will exhibit some necessary conditions on the data already on the level $V_{1}$ that involve both the initial conductances and the values of $h_{k}$. The analogous conditions on all levels $V_{n}$ are also necessary, but since they are expressed in terms of the conductances on $V_{n-1}$, which are derived from the data in a complicated way, we have been unable to write them down explicitly. Nevertheless, we are able to show that there are no "dead ends", in the sense that every resistance network on $\Gamma_{n}$ is the restriction of a point-sensitive regular Dirichlet form on SG. Note that this says more than just that there are no algebraic obstructions to extending from $\Gamma_{n-1}$ to $\Gamma_{n}$, since it involves the analytic property that the conductances tend to infinity in the limit.

In Section 4 we show that the functions in $\mathcal{H}$ already determine the initial conductances (up to a constant multiple) under a seemingly reasonable hypothesis that has the form of a local Harnack inequality. We also give an example when the initial conductances are not determined by $\mathcal{H}$.

Given a compatible sequence of Dirichlet forms $\left\{\mathcal{E}_{n}\right\}$ there exists a limiting Dirichlet form $\mathcal{E}$ on $\mathrm{SG}$. For any function $f$ on $\mathrm{SG}$ the sequence $\mathcal{E}_{n}\left(\left.f\right|_{V_{n}},\left.f\right|_{V_{n}}\right)$ is monotone increasing, so

$$
\mathcal{E}(f, f)=\lim _{n \rightarrow \infty} \mathcal{E}_{n}\left(\left.f\right|_{V_{n}},\left.f\right|_{V_{n}}\right)
$$

is always defined (allowing $+\infty$ ) for a function $f$ on $V_{*}$, and we may define $\operatorname{dom} \mathcal{E}$ as all functions with $\mathcal{E}(f, f)<\infty$. It is not hard to see that $\mathcal{E}_{n}$ is the restriction of $\mathcal{E}$ to $\Gamma_{n}$. In what follows we always assume that the conductances $c(x, y)$ are positive. 
It is evident that $\mathcal{E}$ is point-sensitive, as demonstrated in Proposition 7.1 (Theorem 7.2 gives another proof). Many questions related to such sequences of Dirichlet forms $\mathcal{E}_{n}$ are studied in [Ki4] in detail. An important tool in this study is an effective resistance metric $R$ to be defined in Section 7 . If $\Omega$ is the $R$-completion of $V_{*}$, any function in $\operatorname{dom} \mathcal{E}$ is a restriction of an $R$-continuous function on $\Omega$. We will show in Section 7 that any point of $\Omega$ has positive capacity, and relate it to some properties of Green's function.

In the case when harmonic functions are continuous, $\Omega$ can be naturally considered to be a subset of SG. Under this assumption an important question is whether the harmonic structure is regular, that is $\Omega=\mathrm{SG}$. The answer is positive if all the conductances tend to infinity, but this condition is not necessary, as demonstrated by an example in Section 7 . We also give an example where harmonic functions are continuous but $\Omega \neq \mathrm{SG}$.

Another set of objects naturally associated with $\mathcal{E}$ is the set of energy measures $([\mathbf{K u}])$. For every $f \in \operatorname{dom} \mathcal{E}$ there is a positive measure $\nu_{f}$ such that $\mathcal{E}(f, f)=\nu_{f}(\mathrm{SG})$ and more generally there is a signed measure $\nu_{f, g}$ for each pair $f, g \in \operatorname{dom} \mathcal{E}$ (with $\nu_{f, f}=\nu_{f}$ ) such that

$$
\mathcal{E}(f, g)=\nu_{f, g}(\mathrm{SG}) \text {. }
$$

It is conceivable that it determines $\mathcal{E}$ but we have been unable to prove this.

To put our results into perspective, let us review the situation when the underlying space is the unit interval. Given a Dirichlet form, let $h$ denote the harmonic function satisfying the boundary conditions $h(0)=0, h(1)=1$. The reciprocals of the conductances determine a finite positive measure $\mu$ of total mass $c^{-1}$, where $c$ is the conductance coefficient $c(0,1)$, and $h(x)=$ $c \mu([0, x])$. The only conditions on the measure are that it be continuous and assign positive mass to nontrivial intervals. The only conditions on the function $h$ are that it be continuous and strictly increasing. Note that $\mu$ determines $h$ but $h$ only determines $\mu$ up to a constant multiple. The Dirichlet form is expressible in terms of $c$ and $h$ as

$$
\mathcal{E}(f, f)=c \int_{0}^{1}\left|\frac{d}{d x}\left(f \circ h^{-1}(x)\right)\right|^{2} d x,
$$

with $\operatorname{dom} \mathcal{E}$ consisting of all functions $f$ for which $f \circ h^{-1}$ belongs to the Sobolev space $L_{1}^{2}$. In this case the energy measure for $h$ satisfies $\nu_{h}=c^{2} \mu$.

The reader can find an extensive exposition of the analysis on fractals in $[\mathbf{B}, \mathbf{K i 4}]$. These books also contain lengthy bibliographies.

\section{Basic equations}

We consider graphs $\Gamma_{0}$ and $\Gamma_{1}$ as shown in Figure 2.1. $\Gamma_{0}$ has vertices $q_{1}, q_{2}$, $q_{3}$ with conductances $d_{1}, d_{2}, d_{3}$ on the opposite edge. $\Gamma_{2}$ has an additional 3 vertices $x, y, z$ and 9 edges, with conductances $c_{1}, \ldots, c_{9}$ as shown. The boundary of $\Gamma$ consists of the vertices $q_{1}, q_{2}, q_{3}$. We refer to $c_{2}, c_{5}, c_{8}$ as 

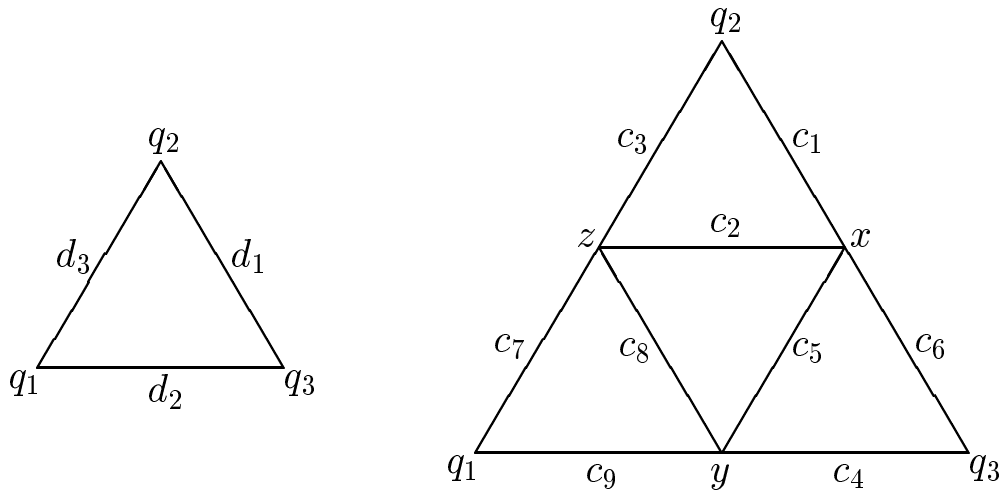

Figure 2.1. The graphs $\Gamma_{0}$ and $\Gamma_{1}$ with vertices and conductances on edges labeled.

inner conductances since the edges do not connect to the boundary vertices, and the others as outer conductances.

We let $h_{j}$ denote the harmonic function on $\Gamma_{1}$ with boundary values $h_{j}\left(q_{k}\right)=\delta_{j k}$. Note that $h_{1}+h_{2}+h_{3}=1$. We write $x_{j}=h_{j}(x), y_{j}=h_{j}(y)$ and $z_{j}=h_{j}(z)$.

The equations that express the fact that $h_{j}$ is harmonic at the 3 vertices $x, y, z$ can be expressed in terms of a matrix $X$ which we write as a sum $X=X_{o}+X_{i}$, with

$$
\begin{aligned}
X_{o} & =\left(\begin{array}{ccc}
c_{1}+c_{6} & 0 & 0 \\
0 & c_{4}+c_{9} & 0 \\
0 & 0 & c_{3}+c_{7}
\end{array}\right), \\
X_{i} & =\left(\begin{array}{ccc}
c_{2}+c_{5} & -c_{5} & -c_{2} \\
-c_{5} & c_{5}+c_{8} & -c_{8} \\
-c_{2} & -c_{8} & c_{2}+c_{8}
\end{array}\right),
\end{aligned}
$$

corresponding to the contributions from the outer and inner conductances, respectively. We also need matrices

$$
H=\left(\begin{array}{lll}
x_{1} & x_{2} & x_{3} \\
y_{1} & y_{2} & y_{3} \\
z_{1} & z_{2} & z_{3}
\end{array}\right)
$$

and

$$
J=\left(\begin{array}{ccc}
0 & c_{1} & c_{6} \\
c_{9} & 0 & c_{4} \\
c_{7} & c_{3} & 0
\end{array}\right)
$$

The harmonic equation is then

$$
X H=J .
$$




\section{Since}

$$
\operatorname{det} J=c_{1} c_{4} c_{7}+c_{3} c_{6} c_{9}
$$

which is positive, it follows that $X$ and $H$ are invertible. It is also clear by inspection that $\operatorname{det} X$ is positive since all terms except $-2 c_{2} c_{5} c_{8}$ are positive, and these cancel $2 c_{2} c_{5} c_{8}$ from the diagonal. A lengthy computation reveals that $\operatorname{det} X$ is given by

$$
\begin{aligned}
c_{1} c_{4} c_{7} & +c_{3} c_{6} c_{9}+c_{1} c_{3}\left(c_{4}+c_{9}\right)+c_{4} c_{6}\left(c_{3}+c_{7}\right)+c_{7} c_{9}\left(c_{1}+c_{6}\right) \\
& +c_{2}\left(c_{4}+c_{9}\right)\left(c_{1}+c_{3}+c_{6}+c_{7}\right)+c_{5}\left(c_{3}+c_{7}\right)\left(c_{1}+c_{4}+c_{6}+c_{9}\right) \\
& +c_{8}\left(c_{1}+c_{6}\right)\left(c_{3}+c_{4}+c_{7}+c_{9}\right) \\
& +\left(c_{2} c_{5}+c_{5} c_{8}+c_{2} c_{8}\right)\left(c_{1}+c_{3}+c_{4}+c_{6}+c_{7}+c_{9}\right) .
\end{aligned}
$$

We can use (2.5) to solve $H=X^{-1} J$ to obtain the harmonic function values explicitly in terms of the conductances, but we will wait until the next section to give the details. Note, however, that (2.5) implies $\operatorname{det} H>0$. Also, the condition $h_{1}+h_{2}+h_{3}=1$ becomes $H 1=1$, where 1 denotes the vector of 1 's.

Another interesting observation from (2.5) is that if we know $H$ and all the outer conductances, we can find all the inner conductances via

$$
X_{i}=-X_{o}+J H^{-1},
$$

since $J$ only involves outer conductances.

Now we consider the consistency condition for the Dirichlet forms on $\Gamma_{1}$ and $\Gamma_{0}$. There are many ways to express this condition, but we concentrate on one that involves only the outer conductances and $H$, namely

$$
\left(\begin{array}{ccc}
d_{2}+d_{3} & -d_{3} & -d_{2} \\
-d_{3} & d_{1}+d_{3} & -d_{1} \\
-d_{2} & -d_{1} & d_{1}+d_{2}
\end{array}\right)=\left(\begin{array}{ccc}
c_{7}+c_{9} & 0 & 0 \\
0 & c_{1}+c_{3} & 0 \\
0 & 0 & c_{4}+c_{6}
\end{array}\right)-J^{*} H
$$

Using the off-diagonal terms, we obtain the set of equations

$$
\left\{\begin{array}{l}
\left(\begin{array}{ll}
x_{3} & z_{3} \\
x_{1} & z_{1}
\end{array}\right)\left(\begin{array}{l}
c_{1} \\
c_{3}
\end{array}\right)=\left(\begin{array}{l}
d_{1} \\
d_{3}
\end{array}\right), \\
\left(\begin{array}{ll}
y_{1} & x_{1} \\
y_{2} & x_{2}
\end{array}\right)\left(\begin{array}{l}
c_{4} \\
c_{6}
\end{array}\right)=\left(\begin{array}{l}
d_{2} \\
d_{1}
\end{array}\right), \\
\left(\begin{array}{ll}
z_{2} & y_{2} \\
z_{3} & y_{3}
\end{array}\right)\left(\begin{array}{l}
c_{7} \\
c_{9}
\end{array}\right)=\left(\begin{array}{l}
d_{3} \\
d_{2}
\end{array}\right) .
\end{array}\right.
$$

We may easily solve (2.10) for the outer conductances, obtaining 
$(2.11)$

$$
\left\{\begin{array}{l}
c_{1}=\frac{\left|\begin{array}{ll}
d_{1} & z_{3} \\
d_{3} & z_{1}
\end{array}\right|}{\left|\begin{array}{ll}
x_{3} & z_{3} \\
x_{1} & z_{1}
\end{array}\right|}, \quad c_{4}=\frac{\left|\begin{array}{ll}
d_{2} & x_{1} \\
d_{1} & x_{2}
\end{array}\right|}{\left|\begin{array}{ll}
y_{1} & x_{1} \\
y_{2} & x_{2}
\end{array}\right|}, \quad c_{7}=\frac{\left|\begin{array}{ll}
d_{3} & y_{2} \\
d_{2} & y_{3}
\end{array}\right|}{\left|\begin{array}{ll}
z_{2} & y_{2} \\
z_{3} & y_{3}
\end{array}\right|}, \\
c_{3}=\frac{\left|\begin{array}{ll}
x_{3} & d_{1} \\
x_{1} & d_{3}
\end{array}\right|}{\left|\begin{array}{ll}
x_{3} & z_{3} \\
x_{1} & z_{1}
\end{array}\right|}, \quad c_{6}=\frac{\left|\begin{array}{ll}
y_{1} & d_{2} \\
y_{2} & d_{1}
\end{array}\right|}{\left|\begin{array}{ll}
z_{2} & d_{3} \\
y_{1} & x_{1} \\
y_{2} & x_{2}
\end{array}\right|}, \quad c_{9}=\frac{\left|\begin{array}{ll}
z_{3} & d_{2}
\end{array}\right|}{\left|\begin{array}{ll}
z_{2} & y_{2} \\
z_{3} & y_{3}
\end{array}\right|} .
\end{array}\right.
$$

In the next section we will show that all the denominators are positive. We then use (2.8) to obtain

$$
\left\{\begin{aligned}
c_{2} & =\left(c_{1}\left|\begin{array}{ll}
x_{1} & x_{3} \\
y_{1} & y_{3}
\end{array}\right|-c_{6}\left|\begin{array}{ll}
x_{1} & x_{2} \\
y_{1} & y_{2}
\end{array}\right|\right) / \operatorname{det} H, \\
c_{5} & =\left(c_{1}\left|\begin{array}{ll}
x_{3} & x_{1} \\
z_{3} & z_{1}
\end{array}\right|-c_{6}\left|\begin{array}{ll}
z_{1} & z_{2} \\
x_{1} & x_{2}
\end{array}\right|\right) / \operatorname{det} H, \\
c_{8} & =\left(c_{9}\left|\begin{array}{ll}
x_{3} & x_{2} \\
y_{3} & y_{2}
\end{array}\right|-c_{4}\left|\begin{array}{ll}
x_{1} & x_{2} \\
y_{1} & y_{2}
\end{array}\right|\right) / \operatorname{det} H .
\end{aligned}\right.
$$

By substituting (2.11) into (2.12) and simplifying we obtain

$$
\left\{\begin{array}{l}
c_{2}=\left|\begin{array}{ccc}
x_{1} & y_{1} & z_{1} \\
d_{1} x_{1} & d_{2} y_{2} & d_{3} z_{3} \\
x_{3} & y_{3} & z_{3}
\end{array}\right| /\left(\left|\begin{array}{ll}
x_{3} & z_{3} \\
x_{1} & z_{1}
\end{array}\right| \operatorname{det} H\right), \\
c_{5}=\left|\begin{array}{ccc}
x_{1} & y_{1} & z_{1} \\
x_{2} & y_{2} & z_{2} \\
d_{1} x_{1} & d_{2} y_{2} & d_{3} z_{3}
\end{array}\right| /\left(\left|\begin{array}{ll}
y_{1} & x_{1} \\
y_{2} & x_{2}
\end{array}\right| \operatorname{det} H\right), \\
c_{8}=\left|\begin{array}{ccc}
d_{1} x_{1} & d_{2} y_{2} & d_{3} z_{3} \\
x_{2} & y_{2} & z_{2} \\
x_{3} & y_{3} & z_{3}
\end{array}\right| /\left(\left|\begin{array}{ll}
z_{2} & y_{2} \\
z_{3} & y_{3}
\end{array}\right| \operatorname{det} H\right) .
\end{array}\right.
$$

We may summarize these computations in the following theorem:

Theorem 2.1. Given the conductances $\left\{d_{j}\right\}$ on $\Gamma_{0}$ and the harmonic values $H$ on $\Gamma_{1}$ satisfying $H 1=1$, there is at most one choice of conductances $\left\{c_{j}\right\}$ on $\Gamma_{1}$ which yields these values, and there is such a choice, with values given by (2.11) and (2.13), if and only if these values are all positive.

Proof. We deduced (2.11) and (2.13) under the assumption that such conductances exist, so this proves uniqueness. Conversely, assume all values 
given in (2.11) and (2.13) are positive. From (2.11) we obtain (2.10). This is equivalent to (2.9) under the assumption $H 1=1$; this yields the diagonal terms. From (2.13) and (2.11) we get (2.12), and also the other 3 expressions for $c_{2}, c_{5}, c_{8}$, which together yield (2.8), or equivalently (2.5). But (2.5) says that $H$ gives the correct values for the harmonic functions determined by the conductances $\left\{c_{j}\right\}$, and then (2.5) and (2.9) tell us that the restriction of the Dirichlet form on $\Gamma_{1}$ to $\Gamma_{0}$ gives the conductances $\left\{d_{j}\right\}$.

To use these expressions iteratively it is useful to put them in a form that is relative to any choice of 3 linearly independent harmonic functions, not just the choice $\left(h_{1}, h_{2}, h_{3}\right)$ made above. Let $\left(\widetilde{h}_{1}, \widetilde{h}_{2}, \widetilde{h}_{3}\right)$ denote such functions. For any vertices $\left(p_{1}, p_{2}, p_{3}\right)$ of $\Gamma_{1}$ define the $3 \times 3$ matrix

$$
\widetilde{h}\left(p_{1}, p_{2}, p_{3}\right)=\left\{\widetilde{h}_{j}\left(p_{k}\right)\right\} .
$$

Corollary 2.2. Suppose $\left(\widetilde{h}_{1}, \widetilde{h}_{2}, \widetilde{h}_{3}\right)$ is any set of 3 linearly independent harmonic functions on $\Gamma_{1}$ for conductances $\left\{c_{j}\right\}$, and suppose the restriction to $\Gamma_{0}$ yields conductances $\left\{d_{j}\right\}$. Then the outer conductances are given by

$$
\left\{\begin{array}{l}
c_{1}=\frac{d_{1} \operatorname{det} \widetilde{h}\left(q_{2}, q_{3}, z\right)+d_{3} \operatorname{det} \widetilde{h}\left(q_{2}, q_{1}, z\right)}{\operatorname{det} \widetilde{h}\left(q_{2}, x, z\right)}, \\
c_{3}=\frac{d_{1} \operatorname{det} \widetilde{h}\left(q_{2}, x, q_{3}\right)+d_{2} \operatorname{det} \widetilde{h}\left(q_{2}, x, q_{1}\right)}{\operatorname{det} \widetilde{h}\left(q_{2}, x, z\right)}, \\
c_{4}=\frac{d_{1} \operatorname{det} \widetilde{h}\left(q_{3}, q_{2}, x\right)+d_{2} \operatorname{det} \widetilde{h}\left(q_{3}, q_{1}, x\right)}{\operatorname{det} \widetilde{h}\left(q_{3}, y, x\right)} \\
c_{6}=\frac{d_{1} \operatorname{det} \widetilde{h}\left(q_{3}, y, q_{2}\right)+d_{2} \operatorname{det} \widetilde{h}\left(q_{3}, y, q_{1}\right)}{\operatorname{det} \widetilde{h}\left(q_{3}, y, x\right)}, \\
c_{7}=\frac{d_{2} \operatorname{det} \widetilde{h}\left(q_{1}, q_{3}, y\right)+d_{3} \operatorname{det} \widetilde{h}\left(q_{1}, q_{2}, y\right)}{\operatorname{det} \widetilde{h}\left(q_{1}, z, y\right)}, \\
c_{9}=\frac{d_{2} \operatorname{det} \widetilde{h}\left(q_{1}, z, q_{3}\right)+d_{3} \operatorname{det} \widetilde{h}\left(q_{1}, z, q_{2}\right)}{\operatorname{det} \widetilde{h}\left(q_{1}, z, y\right)},
\end{array}\right.
$$

and the inner conductances by

$$
\left\{\begin{array}{l}
c_{2}= \\
\frac{d_{1} \operatorname{det} \widetilde{h}\left(x, q_{2}, q_{3}\right) \operatorname{det} \widetilde{h}\left(q_{2}, y, z\right)-d_{2} \operatorname{det} \widetilde{h}\left(q_{1}, y, q_{3}\right) \operatorname{det} \widetilde{h}\left(q_{2}, x, z\right)+d_{3} \operatorname{det} \widetilde{h}\left(q_{1}, q_{2}, z\right) \operatorname{det} \widetilde{h}\left(q_{2}, x, y\right)}{\operatorname{det} \widetilde{h}\left(q_{1}, q_{2}, q_{3}\right) \operatorname{det} \widetilde{h}\left(q_{2}, x, z\right)} \\
c_{5}= \\
\frac{d_{1} \operatorname{det} \widetilde{h}\left(x, q_{2}, q_{3}\right) \operatorname{det} \widetilde{h}\left(q_{3}, y, z\right)+d_{2} \operatorname{det} \widetilde{h}\left(q_{1}, y, q_{3}\right) \operatorname{det} \widetilde{h}\left(q_{3}, x, z\right)-d_{3} \operatorname{det} \widetilde{h}\left(q_{1}, q_{2}, z\right) \operatorname{det} \widetilde{h}\left(q_{3}, y, x\right)}{\operatorname{det} \widetilde{h}\left(q_{1}, q_{2}, q_{3}\right) \operatorname{det} \widetilde{h}\left(q_{3}, y, x\right)} \\
c_{8}= \\
\frac{-d_{1} \operatorname{det} \widetilde{h}\left(x, q_{2}, q_{3}\right) \operatorname{det} \widetilde{h}\left(q_{1}, z, y\right)+d_{2} \operatorname{det} \widetilde{h}\left(q_{1}, y, q_{3}\right) \operatorname{det} \widetilde{h}\left(q_{1}, x, z\right)+d_{3} \operatorname{det} \widetilde{h}\left(q_{1}, q_{2}, z\right) \operatorname{det} \widetilde{h}\left(q_{1}, x, y\right)}{\operatorname{det} \widetilde{h}\left(q_{1}, q_{2}, q_{3}\right) \operatorname{det} \widetilde{h}\left(q_{1}, z, y\right)} .
\end{array}\right.
$$


Proof. First we observe that for the initial choice $\widetilde{h}=h,(2.15)$ is the same as (2.11) and (2.16) is the same as (2.13), so the result is valid in that case. But in general $\widetilde{h}_{j}=\sum_{k} B_{j k} h_{k}$ for some invertible matrix $B$, hence $\widetilde{h}\left(p_{1}, p_{2}, p_{3}\right)=$ $h\left(p_{1}, p_{2}, p_{3}\right) B^{*}$. Thus ratios of quantities of the form $\operatorname{det} \widetilde{h}\left(p_{1}, p_{2}, p_{3}\right)$ are independent of the choice of $\widetilde{h}$, and (2.15) and (2.16) are composed entirely of such ratios.

It is also possible to express these identities in terms of resistances rather than conductances. If we write $R_{j}=d_{j}^{-1}$ and $r_{j}=c_{j}^{-1}$, we have

$$
r_{1}=R_{1} R_{3} \frac{\left|\begin{array}{ll}
x_{3} & z_{3} \\
x_{1} & z_{1}
\end{array}\right|}{\left|\begin{array}{ll}
R_{3} & z_{3} \\
R_{1} & z_{1}
\end{array}\right|}, \quad \text { etc. }
$$

and

$$
r_{2}=R_{1} R_{2} R_{3} \operatorname{det} H \frac{\left|\begin{array}{cc}
x_{3} & z_{3} \\
x_{1} & z_{1}
\end{array}\right|}{}\left|\begin{array}{ccc}
x_{1} & y_{1} & z_{1} \\
R_{2} R_{3} x_{1} & R_{1} R_{3} y_{3} & R_{1} R_{2} z_{3} \\
x_{3} & y_{3} & z_{3}
\end{array}\right|, \text { etc. }
$$

Corollary 2.3. Two Dirichlet forms on SG (giving points positive capacity) having the same harmonic functions and the same initial conductances on $\Gamma_{0}$ must be equal. In particular, the space of Dirichlet forms may be parameterized by a subset of the space of 6 -tuples $\left(d_{1}, d_{2}, d_{3}, h_{1}, h_{2}, h_{3}\right)$ where $d_{j}>0$, and $h_{j}$ are continuous functions $\mathrm{SG} \rightarrow \mathbb{R}$ satisfying $h_{j}\left(q_{k}\right)=\delta_{j k}$ and $h_{1}+h_{2}+h_{3}=1$.

Proof. Applying Theorem 2.1 inductively we see that the two Dirichlet forms have the same conductances at each level, hence by (1.2) they are equal.

In the next section we will find some necessary conditions that the 6 -tuple must satisfy. However, we have not been able to find really explicit necessary and sufficient conditions for a 6-tuple to correspond to a Dirichlet form.

\section{Positivity conditions}

Our first goal is to show that the denominators in (2.11) are all positive. To do this we return to the explicit solution of (2.5) for $H$ in terms of the conductances $\left\{c_{j}\right\}$. In (2.7) we have expressed $\operatorname{det} X$ as a sum of 50 positive terms, each a product of 3 distinct conductances. We may describe all the terms in a scheme that allows three types. There are 8 of type I, a product of 3 outer conductances corresponding to edges in the graph that are disconnected. There are 24 of type II, a product of one inner conductance and 2 outer conductances where one of the outer edges is connected to the 
inner edge, and the other outer edge is not connected to the inner edge. There are 18 of type III, a product of 2 inner and one outer conductance without any other restrictions.

Each entry of $H$ is a ratio with denominator $\operatorname{det} X$ and numerator equal to a sum of terms selected from the 50 terms in $\operatorname{det} X$, as follows:

$$
\left\{\begin{aligned}
x_{1} \operatorname{det} X= & \left(c_{2} c_{5}+c_{2} c_{8}+c_{5} c_{8}\right)\left(c_{7}+c_{9}\right)+c_{7} c_{9}\left(c_{2}+c_{5}\right) \\
& +c_{2} c_{4} c_{7}+c_{3} c_{5} c_{9} \\
y_{1} \operatorname{det} X= & \left(c_{2} c_{5}+c_{2} c_{8}+c_{5} c_{8}\right)\left(c_{7}+c_{9}\right)+c_{7} c_{8}\left(c_{1}+c_{6}\right) \\
& +\left(c_{3}+c_{7}\right) c_{9}\left(c_{2}+c_{5}\right)+c_{9}\left(c_{1}+c_{6}\right)\left(c_{2}+c_{3}+c_{7}+c_{8}\right) \\
z_{1} \operatorname{det} X= & \left(c_{2} c_{5}+c_{2} c_{8}+c_{5} c_{8}\right)\left(c_{7}+c_{9}\right)+c_{9} c_{8}\left(c_{1}+c_{6}\right) \\
& +\left(c_{4}+c_{9}\right) c_{7}\left(c_{2}+c_{5}\right)+c_{7}\left(c_{1}+c_{6}\right)\left(c_{4}+c_{5}+c_{8}+c_{9}\right) \\
x_{2} \operatorname{det} X= & \left(c_{2} c_{5}+c_{2} c_{8}+c_{5} c_{8}\right)\left(c_{1}+c_{3}\right)+c_{2} c_{3}\left(c_{4}+c_{9}\right) \\
& +c_{1}\left(c_{3}+c_{7}\right)\left(c_{5}+c_{8}\right)+c_{1}\left(c_{4}+c_{9}\right)\left(c_{2}+c_{3}+c_{7}+c_{8}\right) \\
y_{2} \operatorname{det} X= & \left(c_{2} c_{5}+c_{2} c_{8}+c_{5} c_{8}\right)\left(c_{1}+c_{3}\right)+c_{1} c_{3}\left(c_{5}+c_{8}\right) \\
& +c_{1} c_{5} c_{7}+c_{3} c_{6} c_{8} \\
z_{2} \operatorname{det} X= & \left(c_{2} c_{5}+c_{2} c_{8}+c_{5} c_{8}\right)\left(c_{1}+c_{3}\right)+c_{1} c_{2}\left(c_{4}+c_{9}\right) \\
& +c_{3}\left(c_{1}+c_{6}\right)\left(c_{5}+c_{8}\right)+c_{3}\left(c_{4}+c_{9}\right)\left(c_{1}+c_{2}+c_{5}+c_{6}\right) \\
x_{3} \operatorname{det} X= & \left(c_{2} c_{5}+c_{2} c_{8}+c_{5} c_{8}\right)\left(c_{4}+c_{6}\right)+c_{4} c_{5}\left(c_{3}+c_{7}\right) \\
& +c_{6}\left(c_{4}+c_{9}\right)\left(c_{2}+c_{8}\right)+c_{6}\left(c_{3}+c_{7}\right)\left(c_{4}+c_{5}+c_{8}+c_{9}\right) \\
y_{3} \operatorname{det} X= & \left(c_{2} c_{5}+c_{2} c_{8}+c_{5} c_{8}\right)\left(c_{4}+c_{6}\right)+c_{5} c_{6}\left(c_{3}+c_{7}\right) \\
& +c_{4}\left(c_{1}+c_{6}\right)\left(c_{2}+c_{8}\right)+c_{4}\left(c_{3}+c_{7}\right)\left(c_{1}+c_{2}+c_{4}+c_{5}\right) \\
z_{3} \operatorname{det} X= & \left(c_{2} c_{5}+c_{2} c_{8}+c_{5} c_{8}\right)\left(c_{4}+c_{6}\right)+c_{4} c_{6}\left(c_{2}+c_{8}\right) \\
& +c_{2} c_{6} c_{9}+c_{1} c_{4} c_{8} .
\end{aligned}\right.
$$

Note that $x_{1} \operatorname{det} X, y_{2} \operatorname{det} X$ and $z_{3} \operatorname{det} X$ consist of 10 terms each, while the others consist of 20 terms.

Theorem 3.1. Each of the determinants $\left|\begin{array}{ll}y_{1} & x_{1} \\ y_{2} & x_{2}\end{array}\right|,\left|\begin{array}{ll}z_{2} & y_{2} \\ z_{3} & y_{3}\end{array}\right|$ and $\left|\begin{array}{ll}x_{3} & z_{3} \\ x_{1} & z_{1}\end{array}\right|$ is
positive.

Proof. We give two proofs. The first is computational, while the second is conceptual. For the first proof we show

$$
\left(y_{1} \operatorname{det} X\right)\left(x_{2} \operatorname{det} X\right)-\left(y_{2} \operatorname{det} X\right)\left(x_{1} \operatorname{det} X\right)>0
$$

by showing that it is a sum of 300 positive terms. Note that the first product consists of 400 terms and the second product consists of 100 terms. We claim that all 100 terms in the second product are contained among the 400 terms of the first product. In fact, 9 of the 10 terms in $x_{1} \operatorname{det} X$, namely all except $c_{2} c_{4} c_{7}$, are among the 20 terms of $y_{1} \operatorname{det} X$. Similarly, 9 of the 10 terms in $y_{2} \operatorname{det} X$, all except $c_{3} c_{6} c_{8}$, are among the 20 terms of $x_{2} \operatorname{det} X$. This already takes care of 81 terms of the product. It remains to check 
the 19 terms of the product that have either $c_{3} c_{6} c_{8}$ or $c_{2} c_{4} c_{7}$ as one of the factors. This may be seen by inspection. For example, $\left(c_{3} c_{6} c_{8}\right)\left(c_{2} c_{4} c_{7}\right)$ appears as $\left(c_{2} c_{3} c_{4}\right)\left(c_{6} c_{7} c_{8}\right)$, and $\left(c_{3} c_{6} c_{8}\right)\left(c_{2} c_{7} c_{9}\right)$ appears in 2 different ways, $\left(c_{2} c_{3} c_{9}\right)\left(c_{6} c_{7} c_{8}\right)$ or $\left(c_{2} c_{3} c_{8}\right)\left(c_{6} c_{7} c_{9}\right)$. The positivity of the other 2 determinants follows by symmetry.

The second proof is based on the following interpretation of the result. Note that

$$
\left|\begin{array}{ll}
y_{1} & x_{1} \\
y_{2} & x_{2}
\end{array}\right|=\left|\begin{array}{lll}
y_{1} & x_{1} & 0 \\
y_{1} & x_{2} & 0 \\
y_{3} & x_{3} & 1
\end{array}\right|=\operatorname{det} h\left(y, x, q_{3}\right)
$$

and $\left(y, x, q_{3}\right)$ are the vertices of a small triangle in $\Gamma_{1}$ traversed in the same order as $\left(q_{1}, q_{2}, q_{3}\right)$ traverses the large triangle. Since the determinant is a continuous function of the conductances, and it is clearly positive for the choice of equal conductances $\left(y_{1}=x_{2}=\frac{2}{5}, y_{2}=x_{1}=\frac{1}{5}\right)$, it suffices to show that it is never zero. The determinant being zero means that there is a nonzero harmonic function whose restriction to the points $\left(x, y, q_{3}\right)$ is zero. We now show that this is impossible.

Suppose $h$ is a harmonic function on $\Gamma_{1}$ satisfying $h(y)=h(x)=h\left(q_{3}\right)=0$. If $h$ is not identically zero we may arrange to have $h\left(q_{1}\right)>0$, without loss of generality. Since $h$ is harmonic at $y$ we must have $h(z)<0$. Since $h$ is harmonic at $x$ we then must have $h\left(q_{2}\right)>0$. But then $h$ will not be harmonic at $z$.

The unique extension property for harmonic functions from small triangles proved above is not usually valid for graphs that arise in the study of self-similar fractals. It fails for the pentagasket ([ASST]) and hexagasket fractals, for examples.

The next result is a relativized version of the result, analogous to Corollary 2.2. Note that $h\left(q_{1}, q_{2}, q_{3}\right)$ is the identity matrix so $\operatorname{det} h\left(q_{1}, q_{2}, q_{3}\right)=1$.

Corollary 3.2. Let $\left(\widetilde{h}_{1}, \widetilde{h}_{2}, \widetilde{h}_{3}\right)$ be linearly independent harmonic functions for which $\operatorname{det} \widetilde{h}\left(q_{1}, q_{2}, q_{3}\right)>0$. Then $\operatorname{det} \widetilde{h}\left(y, x, q_{3}\right)$, $\operatorname{det} \widetilde{h}\left(z, q_{2}, x\right)$ and $\operatorname{det} \widetilde{h}\left(q_{1}, z, y\right)$ are all positive.

Proof. As in the proof of Corollary 2.2 we write $\widetilde{h}=h B^{*}$. Since each matrix is multiplied by $B^{*}$, we must have $\operatorname{det} B^{*}>0$ from the hypothesis. Then multiplication by $B^{*}$ preserves the sign of the other determinants.

Since the denominators in (2.11) are all positive, so are the numerators. These inequalities may be summarized as

$$
\frac{z_{1}}{z_{3}}>\frac{d_{3}}{d_{1}}>\frac{x_{1}}{x_{3}}, \quad \frac{x_{2}}{x_{1}}>\frac{d_{1}}{d_{2}}>\frac{y_{2}}{y_{1}}, \quad \frac{y_{3}}{y_{2}}>\frac{d_{2}}{d_{3}}>\frac{z_{3}}{z_{2}} .
$$


Note that the positivity of the determinants in Theorem 3.1 means that the inequalities in (3.3) are valid without the middle term. Also, by multiplying the 3 rows of (3.3) the middle term becomes 1, and we obtain

$$
x_{1} y_{2} z_{3}<x_{2} y_{3} z_{1}, \quad x_{1} y_{2} z_{3}<x_{3} y_{1} z_{2} .
$$

It would also be possible to give a computational proof of (3.4) along the lines of the first proof of Theorem 3.1.

Theorem 3.3. Given positive conductances $\left\{d_{j}\right\}$ and a matrix $H$ with positive entries satisfying $H 1=1$, a necessary and sufficient condition for the existence of positive conductances $\left\{c_{j}\right\}$ such that $H$ is the matrix of harmonic values and $\left\{d_{j}\right\}$ is the restriction to $\Gamma_{0}$ is that the following 13 determinants all be positive:

$$
\begin{gathered}
\operatorname{det} H, \\
\left|\begin{array}{ll}
y_{1} & x_{1} \\
y_{2} & x_{2}
\end{array}\right|,\left|\begin{array}{ll}
z_{2} & y_{2} \\
z_{3} & y_{3}
\end{array}\right|,\left|\begin{array}{ll}
x_{3} & z_{3} \\
x_{1} & z_{1}
\end{array}\right|, \\
\left|\begin{array}{ll}
d_{1} & z_{3} \\
d_{3} & z_{1}
\end{array}\right|,\left|\begin{array}{cc}
x_{3} & d_{1} \\
x_{1} & d_{3}
\end{array}\right|,\left|\begin{array}{cc}
d_{2} & x_{1} \\
d_{1} & x_{2}
\end{array}\right|,\left|\begin{array}{ll}
y_{1} & d_{2} \\
y_{2} & d_{1}
\end{array}\right|,\left|\begin{array}{cc}
d_{3} & y_{2} \\
d_{2} & y_{3}
\end{array}\right|,\left|\begin{array}{cc}
z_{2} & d_{3} \\
z_{3} & d_{2}
\end{array}\right|, \\
\left|\begin{array}{ccc}
x_{1} & y_{1} & z_{1} \\
x_{2} & y_{2} & z_{2} \\
d_{1} x_{1} & d_{2} y_{2} & d_{3} z_{3}
\end{array}\right|,\left|\begin{array}{ccc}
x_{1} & y_{1} & z_{1} \\
d_{1} x_{1} & d_{2} y_{2} & d_{3} z_{3} \\
x_{3} & y_{3} & z_{3}
\end{array}\right|,\left|\begin{array}{ccc}
d_{1} x_{1} & d_{2} y_{2} & d_{3} z_{3} \\
x_{2} & y_{2} & z_{2} \\
x_{3} & y_{3} & z_{3}
\end{array}\right| .
\end{gathered}
$$

Proof. We have already observed the necessity of the positivity of (3.5), (3.6) and (3.7), and the condition $H 1=1$. The positivity of the inner conductances (2.13) then requires the positivity of (3.8). Conversely, if all 13 determinants are positive then (2.11) and (2.13) show that all the conductances $\left\{c_{j}\right\}$ are positive, and the result follows from Theorem 2.1.

We now turn to the problem of characterizing all possible matrices $H$ of harmonic values. This is just the problem of finding all $H$ such that there exists a choice of $\left\{d_{j}\right\}$ so that the 13 determinants are positive.

Theorem 3.4. Given a matrix $H$ with positive entries satisfying $H 1=1$, there exists a set $\left\{c_{j}\right\}$ of positive conductances on $\Gamma_{1}$ having $H$ as its matrix of harmonic values, if and only if the 4 determinants (3.5) and (3.6) are positive, and there exists an open half-space in $\mathbb{R}^{3}$ containing all 12 of the following vectors:

$$
(1,0,0),(0,1,0),(0,0,1),
$$

(3.10) $\left(x_{1}, y_{1}, z_{1}\right) \times\left(x_{2}, y_{2}, z_{2}\right),\left(x_{2}, y_{2}, z_{2}\right) \times\left(x_{3}, y_{3}, z_{3}\right),\left(x_{3}, y_{3}, z_{3}\right) \times\left(x_{1}, y_{1}, z_{1}\right)$,

(3.11) $(0,1,0) \times\left(x_{1}, y_{1}, z_{1}\right),(0,0,1) \times\left(x_{2}, y_{2}, z_{2}\right),(1,0,0) \times\left(x_{3}, y_{3}, z_{3}\right)$,

$$
\left(x_{1}, y_{1}, z_{1}\right) \times(0,0,1),\left(x_{2}, y_{2}, z_{2}\right) \times(1,0,0),\left(x_{3}, y_{3}, z_{3}\right) \times(0,1,0) .
$$


Moreover, if these conditions hold, $\left(d_{1}, d_{2}, d_{3}\right)$ may be chosen so that the vector $\left(d_{1} x_{1}, d_{2} y_{2}, d_{3} z_{3}\right)$ has positive inner product with all 12 of the vectors above.

Proof. By choosing $\left\{d_{j}\right\}$ appropriately, we can make the vector

$$
v=\left(d_{1} x_{1}, d_{2} y_{2}, d_{3} z_{3}\right)
$$

equal to any vector with positive entries. This means the inner products of $v$ with the vectors (3.9) are all positive. The positivity of the determinants (3.8) is clearly equivalent to the positivity of the inner product of $v$ and the vectors (3.10). We claim the same equivalence with (3.7) and (3.11). For example,

$$
\left|\begin{array}{ll}
d_{1} & z_{3} \\
d_{3} & z_{1}
\end{array}\right|>0 \Longleftrightarrow\left(d_{1} x_{1}\right) z_{1}-\left(d_{3} z_{3}\right) x_{1}>0
$$

and the left-hand side of this last inequality equals $v \cdot\left((0,1,0) \times\left(x_{1}, y_{1}, z_{1}\right)\right)$. The other equivalences follow similarly. The result now follows from the observation that the existence of an open half-space $\{u: u \cdot v>0\}$ containing a set of vectors is equivalent to the existence of a vector with positive inner product with all of them.

The next result shows that we will never hit a "dead end" in extending a Dirichlet form from $\Gamma_{n}$ to SG.

Theorem 3.5. Every resistance network on $\Gamma_{n-1}$ is the restriction of a Dirichlet form on SG which is a self-similar regular Dirichlet form on every small triangle in $\Gamma_{n}$.

Proof. It suffices to give a proof for $\Gamma_{0}$. Let positive conductances $d_{1}, d_{2}, d_{3}$ be fixed. We will show that, for any small enough $\varepsilon>0$, the network is the restriction of a network on $\Gamma_{1}$ such that $c_{9}=c_{7}=\varepsilon c_{8}=1 / s_{1}$, $c_{1}=c_{3}=\varepsilon c_{2}=1 / s_{2}$, and $c_{4}=c_{6}=\varepsilon c_{5}=1 / s_{3}$.

Let $R_{j}$ be the effective resistances in the network $\Gamma_{0}$ and let $F_{j}\left(s_{1}, s_{2}, s_{3}, \varepsilon\right)$ be the effective resistances between points in $V_{0}$ in the network $\Gamma_{1}$. We have to show that the equations $R_{j}=F_{j}\left(s_{1}, s_{2}, s_{3}, \varepsilon\right), j=1,2,3$, have positive solutions $s_{1}, s_{2}, s_{3}$ for all small enough $\varepsilon>0$.

Note that we can take $\varepsilon=0$, since in this case we have a well-defined network with zero inner resistances. Considering all indices mod 3 , we have

$$
F_{j}\left(s_{1}, s_{2}, s_{3}, 0\right)=\frac{1}{2}\left(s_{j-1}+s_{j+1}\right) .
$$

It is easy to see that if $\varepsilon=0$ then the equations have unique positive solutions $s_{1}, s_{2}, s_{3}$ since $R_{1}, R_{2}, R_{3}$ satisfy the triangle inequalities. One can also see that the $3 \times 3$ matrix

$$
\left\{\frac{\partial F_{j}}{\partial s_{i}}\left(s_{1}, s_{2}, s_{3}, 0\right)\right\}_{i, j=1,2,3}
$$


is nonsingular. The functions $F_{j}$ are rational, and so for any small enough $\varepsilon>0$, positive solutions exist by the implicit function theorem and the continuity of the derivatives.

Then each of the three triangles in $\Gamma_{1}$ has conductances proportional to $(1,1,1 / \varepsilon)$. Each such triangle of conductances is the restriction of a regular self-similar Dirichlet form for any $\varepsilon>0$, as shown in [Sa] (see also [Ki4], Exercise 3.1).

\section{Uniqueness of the initial conductances}

We now show that, under certain assumptions, the initial conductances $d_{1}, d_{2}, d_{3}$ are uniquely determined up to a constant multiple by the space of harmonic functions $\mathcal{H}$. These assumptions have the form of local Harnack inequalities at the boundary points. In particular, if $\mathcal{H}$ corresponds to a self-similar harmonic structure then the initial conductances are determined uniquely up to a constant multiple.

Let $z^{(n)}=F_{1}^{n}\left(q_{2}\right)$ and $y^{(n)}=F_{1}^{n}\left(q_{3}\right)$, in particular $z=z^{(1)}$ and $y=y^{(1)}$. Also let $z_{j}^{(n)}=h_{j}\left(z^{(n)}\right)$ and $y_{j}^{(n)}=h_{j}\left(y^{(n)}\right)$.

Lemma 4.1 (monotonicity). For any $n \geq 0$,

$$
\frac{z_{3}^{(n-1)}}{z_{2}^{(n-1)}}<\frac{z_{3}^{(n)}}{z_{2}^{(n)}}<\frac{d_{2}}{d_{3}}<\frac{y_{3}^{(n)}}{y_{2}^{(n)}}<\frac{y_{3}^{(n-1)}}{y_{2}^{(n-1)}} .
$$

Proof. Let

$$
M^{(n)}=\left(\begin{array}{ll}
z_{2}^{(n)} & y_{2}^{(n)} \\
z_{3}^{(n)} & y_{3}^{(n)}
\end{array}\right) .
$$

One can show that for any $m \geq 0$ we have $\operatorname{det} M^{(n)}>0$ and

$$
\frac{z_{3}^{(m)}}{z_{2}^{(m)}}<\frac{d_{2}}{d_{3}}<\frac{y_{3}^{(m)}}{y_{2}^{(m)}} .
$$

The argument is similar to (3.3) because $M^{(n)}$ satisfies a relation analogous to $(2.10)$. Set

$$
A_{n}=\left(\begin{array}{cc}
a_{11}^{(n)} & a_{12}^{(n)} \\
a_{21}^{(n)} & a_{22}^{(n)}
\end{array}\right)=\left(M^{(n-1)}\right)^{-1} M^{(n)} .
$$

It is clear from the definition that, for a harmonic function $h$ with $h\left(q_{1}\right)=0$, $A_{n}^{T}$ transforms the values of $h$ at $z^{(n-1)}, y^{(n-1)}$ into its values at $z^{(n)}, y^{(n)}$. That is,

$$
\left(\begin{array}{l}
h\left(z^{(n)}\right) \\
h\left(y^{(n)}\right)
\end{array}\right)=\left(\begin{array}{ll}
a_{11}^{(n)} & a_{21}^{(n)} \\
a_{12}^{(n)} & a_{22}^{(n)}
\end{array}\right)\left(\begin{array}{l}
h\left(z^{(n-1)}\right) \\
h\left(y^{(n-1)}\right)
\end{array}\right) .
$$


Hence $a_{n}, b_{n}, c_{n}, d_{n}$ are all positive. Then the required inequalities

$$
\frac{z_{3}^{(n-1)}}{z_{2}^{(n-1)}}<\frac{z_{3}^{(n)}}{z_{2}^{(n)}} \quad \text { and } \quad \frac{y_{3}^{(n)}}{y_{2}^{(n)}}<\frac{y_{3}^{(n-1)}}{y_{2}^{(n-1)}}
$$

are easy to check by induction since $M^{(n)}=M^{(n-1)} A_{n}$. Together, (4.2) and (4.4) yield (4.1).

Corollary 4.2. If

$$
\lim _{n \rightarrow \infty} \frac{y_{3}^{(n)}}{y_{2}^{(n)}}-\frac{z_{3}^{(n)}}{z_{2}^{(n)}}=0
$$

then $\frac{d_{2}}{d_{3}}$ is uniquely determined and

$$
\lim _{n \rightarrow \infty} \frac{y_{3}^{(n)}}{y_{2}^{(n)}}=\lim _{n \rightarrow \infty} \frac{z_{3}^{(n)}}{z_{2}^{(n)}}=\frac{d_{2}}{d_{3}} .
$$

Theorem 4.3. Suppose the following local form of the Harnack inequality at $q_{1}$ holds: there exists $\delta>0$ such that if $h$ is harmonic and satisfies

$$
h\left(q_{1}\right)=0, h\left(y^{(n-1)}\right)>0, \text { and } h\left(z^{(n-1)}\right)>0,
$$

then

$$
\delta<\frac{h\left(z^{(n)}\right)}{h\left(y^{(n)}\right)}<\frac{1}{\delta}
$$

for all $n \geq 0$. Then the ratio $\frac{d_{3}}{d_{2}}$ is uniquely determined by the space $\mathcal{H}$.

Proof. Our aim is to show that Corollary 4.2 applies. Let $M^{(n)}$ and $A_{n}$ be as defined in the proof of Lemma 4.1. Then it is easy to see that both $a_{11}^{(n)} / a_{12}^{(n)}$ and $a_{21}^{(n)} / a_{22}^{(n)}$ are in $(\delta, 1 / \delta)$. Then

$$
\text { (4.8) } \begin{aligned}
\frac{y_{3}^{(n)}}{y_{2}^{(n)}}-\frac{z_{3}^{(n)}}{z_{2}^{(n)}} & =\frac{\operatorname{det} M^{(n)}}{y_{2}^{(n)} z_{2}^{(n)}} \\
& =\frac{\operatorname{det} M^{(n-1)} \operatorname{det} A_{n}}{\left(z_{2}^{(n-1)} a_{11}^{(n)}+y_{2}^{(n-1)} a_{21}^{(n)}\right)\left(z_{2}^{(n-1)} a_{12}^{(n)}+y_{2}^{(n-1)} a_{22}^{(n)}\right)} \\
& \leq \frac{\operatorname{det} M^{(n-1)}}{z_{2}^{(n-1)} y_{2}^{(n-1)}} \frac{\operatorname{det} A_{n}}{a_{11}^{(n)} a_{22}^{(n)}} \leq\left(\frac{y_{3}^{(n-1)}}{y_{2}^{(n-1)}}-\frac{z_{3}^{(n-1)}}{z_{2}^{(n-1)}}\right)\left(1-\delta^{2}\right),
\end{aligned}
$$

which implies (4.6).

Example 4.4. We will now see that the space of harmonic functions need not determine the initial conductances uniquely. The harmonic functions in this example are not continuous, however. On each level $n$ all the conductances are positive, but some tend to zero as $n \rightarrow \infty$. 
Let

If $H=H_{\varepsilon}$ then, as $\varepsilon \rightarrow 0$,

$$
H_{\varepsilon}=\left(\begin{array}{ccc}
\varepsilon^{2} & \varepsilon-\varepsilon^{2} & 1-\varepsilon \\
1-\varepsilon & \varepsilon^{2} & \varepsilon-\varepsilon^{2} \\
\varepsilon-\varepsilon^{2} & 1-\varepsilon & \varepsilon^{2}
\end{array}\right) .
$$

$$
\begin{aligned}
c_{1} & =d_{1}+d_{1} O(\varepsilon)+d_{3} O(\varepsilon), \\
c_{3} & =\frac{1}{\varepsilon} d_{3}+d_{1} O(1)+d_{3} O(1), \\
c_{2} & =\varepsilon d_{3}+d_{1} O\left(\varepsilon^{2}\right)+d_{2} O\left(\varepsilon^{2}\right)+d_{3} O\left(\varepsilon^{2}\right) .
\end{aligned}
$$

There are similar formulas for the other conductances since $H_{\varepsilon}$ is rotationally invariant. One can see that there exist $K, K^{\prime}>0$ such that if

$$
\max _{i, j=1,2,3} \frac{d_{i}}{d_{j}}<\frac{K}{\varepsilon}
$$

then

$$
\max _{k, \ell=1,2,3} \frac{c_{k}}{c_{\ell}}<\frac{K^{\prime}}{\varepsilon^{2}} .
$$

We suppose that for each $w \in W_{n}$ the matrix $H_{w}$ that defines the continuation of a harmonic function from $F_{w}\left\{q_{1}, q_{2}, q_{3}\right\}$ to $F_{w}\{x, y, z\}$ is $H_{\varepsilon_{n}}$, where the $\varepsilon_{n}>0$ are to be chosen later. In terms of (2.14) this means that

$$
H_{w}=h\left(F_{w}(x), F_{w}(y), F_{w}(z)\right)\left(h\left(F_{w}\left(q_{1}\right), F_{w}\left(q_{2}\right), F_{w}\left(q_{3}\right)\right)\right)^{-1}=H_{\varepsilon_{n}} .
$$

Then all the conductances for all $n$ are positive if (4.9) is satisfied with small enough $\varepsilon=\varepsilon_{0}$ and $\varepsilon_{n+1} \leq K \varepsilon_{n}^{2} / K^{\prime}$ for all $n \geq 0$.

\section{A parameterization of compatible Dirichlet forms}

In this section we describe the compatibility conditions in going from conductances on $\Gamma_{0}$ to conductances on $\Gamma_{1}$ in parametric form. Specifically, for each choice of conductances $\left\{d_{j}\right\}$ we give an algorithm that constructs all compatible Dirichlet forms on $\Gamma_{1}$ based on the choice of 6 parameters chosen without restriction from $(0,1)^{6}$. The correspondence between parameters and Dirichlet forms is one-to-one. We use the $\Delta-Y$ transform and its inverse $([\mathbf{K i} \mathbf{i}])$ several times.

Algorithm 5.1. Given any positive conductances $\left\{d_{j}\right\}$ on $\Gamma_{0}$, for each choice of $\left(\alpha_{1}, \alpha_{2}, \alpha_{3}, \beta_{1}, \beta_{2}, \beta_{3}\right) \in(0,1)^{6}$ perform the following steps to obtain a distinct compatible Dirichlet form on $\Gamma_{1}$ :

Step 1. Compute initial resistances $R_{1}, R_{2}, R_{3}>0$ for the $\Delta-Y$ transformed triangle in Figure 5.1, so that

$$
R_{j}=\frac{d_{j}}{d_{1} d_{2}+d_{2} d_{3}+d_{1} d_{3}} \quad \text { and } \quad d_{j}=\frac{R_{j-1} R_{j+1}}{R_{1}+R_{2}+R_{3}} .
$$


Step 1. Initial network


Step 2.
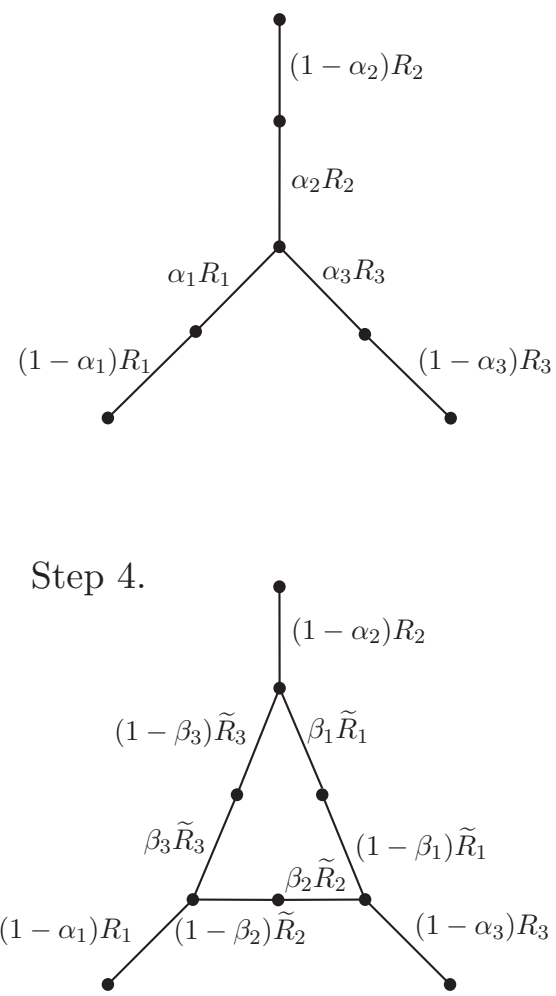

Figure 5.1. Pictorial description of Steps 1-4.

For convenience we identify indices $\bmod 3$, so $R_{0}=R_{3}$, etc.

Step 2. Choose any three numbers $\alpha_{1}, \alpha_{2}, \alpha_{3} \in(0,1)$ to split the resistances $R_{j}$ into $\alpha_{j} R_{j}$ and $\left(1-\alpha_{j}\right) R_{j}$.

Step 3. Do a $Y-\Delta$ transform for resistances $\left(\alpha_{1} R_{1}, \alpha_{2} R_{2}, \alpha_{3} R_{3}\right)$ :

$$
\widetilde{R}_{j}=\frac{\alpha_{1} \alpha_{2} R_{1} R_{2}+\alpha_{1} \alpha_{3} R_{1} R_{3}+\alpha_{2} \alpha_{3} R_{2} R_{3}}{\alpha_{j} R_{j}} .
$$

Step 4. Choose any three numbers $\beta_{1}, \beta_{2}, \beta_{3} \in(0,1)$ to split the resistances $\widetilde{R}_{1}, \widetilde{R}_{2}, \widetilde{R}_{3}$. Then for each $j=1,2,3$ do a $Y-\Delta$ transform on the triple of resistances

$$
\left(R_{1}^{j}, R_{2}^{j}, R_{3}^{j}\right)=\left(\left(1-\alpha_{j}\right) R_{j},\left(1-\beta_{j+1}\right) \widetilde{R}_{j+1}, \beta_{j-1} \widetilde{R}_{j-1}\right) .
$$

These 4 steps are summarized in Figure 5.1. The results give resistances on $\Gamma_{1}$, whose reciprocals define the compatible Dirichlet form. 
Remarks. To simplify computations in this algorithm one can consider the resistances $R_{j}$ as the initial data and avoid the $Y-\Delta$ transform in Step 4 .

The algorithm can easily yield an explicit but complicated formula for the conductances $c_{1}, \ldots, c_{9}$ as a rational function of the initial conductances and the parameters. The points where $\widetilde{R}_{j}$ are divided into $\beta_{j} \widetilde{R}_{j}$ and $\left(1-\beta_{j}\right) \widetilde{R}_{j}$ are the points $x, y, z$. Thus we can also recover the values of the harmonic functions at $x, y, z$, although this is not necessary for the algorithm.

To obtain a parametric description of all compatible Dirichlet forms on $\Gamma_{k}$ we just use the algorithm iteratively on each of the triangles on each level up to $k$; the choices of the 6 parameters can be made independently.

Corollary 5.2. The space of all Dirichlet forms on $\Gamma_{1}$ compatible with a fixed Dirichlet form on $\Gamma_{0}$ is a manifold of dimension 6. More generally, the space of all Dirichlet forms on $\Gamma_{k}$ compatible with a fixed Dirichlet form on $\Gamma_{0}$ is a manifold of dimension $3\left(3^{k}-1\right)$.

Given any choice of the 6 parameters $\alpha_{1}^{w}, \alpha_{2}^{w}, \alpha_{3}^{w}, \beta_{1}^{w}, \beta_{2}^{w}, \beta_{3}^{w} \in(0,1)^{6}$ for each word $w$ of finite length, we obtain a compatible sequence of Dirichlet forms on $\left\{\Gamma_{n}\right\}$. This yields a Dirichlet form on SG with all points having positive capacity exactly when all the conductances tend to infinity (resistances tend to 0). We have not been able to find necessary and sufficient conditions on the parameters for this, but we will give some related results in Section 7 and we can give sufficient conditions for harmonic functions to be continuous.

Next we will show that harmonic functions are continuous if the parameters are uniformly separated from 0 and 1 , and if the parameters are chosen at random. First we need the following lemmas:

Lemma 5.3. Let $M(h)=\max _{x \in \mathrm{SG}} h(x)-\min _{x \in \mathrm{SG}} h(x)$ for a harmonic function $h$. Then, for any $j=1,2,3$,

$$
M\left(h \circ F_{j}\right) \leq(1-m) M(h),
$$

where $m$ is the minimum of the off-diagonal elements of matrix $H$.

Proof. By the maximum principle $M(h)=\max _{i=1,2,3} h\left(q_{i}\right)-\min _{i=1,2,3} h\left(q_{i}\right)$. It is enough to give a proof in the case $j=1$ and $h\left(q_{1}\right)=0$. Then we have

$$
\left(y_{2}+y_{3}\right) \min _{i=1,2,3} h\left(q_{i}\right) \leq h(y) \leq\left(y_{2}+y_{3}\right) \max _{i=1,2,3} h\left(q_{i}\right)
$$

and $y_{2}+y_{3}=1-y_{1} \leq 1-m$. Therefore

$$
(1-m) \min _{i=1,2,3} h\left(q_{i}\right) \leq h(y) \leq(1-m) \max _{i=1,2,3} h\left(q_{i}\right),
$$

since $\min _{i=1,2,3} h\left(q_{i}\right) \leq 0$ and $\max _{i=1,2,3} h\left(q_{i}\right) \geq 0$. A similar estimate holds for $h(z)$, and the result follows.

Lemma 5.4. Let $\delta=\delta\left(\alpha_{1}, \cdots, \beta_{3}\right)=\min _{i=1,2,3} \min \left\{\alpha_{i} \beta_{i+1}, \alpha_{i}\left(1-\beta_{i-1}\right)\right\}$. Then $\delta \leq m$. 
Proof. Since $h_{1} \geq 0$, we have $z_{1}=h_{1}(z) \geq \alpha_{1}\left(1-\beta_{3}\right)$. Similarly $y_{1} \geq \alpha_{1} \beta_{2}$ etc. One can obtain these inequalities, for example, from harmonic equations $h_{1}\left(a_{1}\right)=\alpha_{1}+h(a)\left(1-\alpha_{1}\right)$ and $h_{1}(z)=\left(1-\beta_{3}\right) h\left(a_{1}\right)+\beta_{3} h\left(a_{2}\right)$, where $a, a_{1}, \ldots, a_{3}$ are defined in Section 6 .

We will denote $\delta\left(\alpha_{1}^{w_{1} \cdots w_{k}}, \ldots, \beta_{3}^{w_{1} \cdots w_{k}}\right)$ by $\delta_{w_{1} \cdots w_{k}}$.

\section{Theorem 5.5.}

(1) If for any infinite word $w_{1}, w_{2}, \ldots$

$$
\sum_{k=0}^{\infty} \delta_{w_{1} \cdots w_{k}}=\infty
$$

then harmonic functions are continuous.

(2) Suppose

$$
\delta_{\text {inf }}=\inf _{w \in W_{*}} \delta_{w}>0 .
$$

Then harmonic functions are Hölder continuous with Hölder exponent $1-\delta_{\text {inf }}$.

Proof. By (5.4) and Lemma 5.3 we have, for any finite word $w=w_{1} \cdots w_{n}$,

$$
M\left(h \circ F_{w}\right) \leq M(h) \prod_{k=0}^{n-1}\left(1-\delta_{w_{1} \cdots w_{k}}\right) .
$$

Strictly speaking, the algorithm above defines harmonic functions on $V_{*}$ only. However, the continuity on $V_{*}$ implies the existence of a unique continuation to SG.

\section{Corollary 5.6.}

(1) Suppose that $\xi_{w}=\left(\alpha_{1}^{w}, \alpha_{2}^{w}, \alpha_{3}^{w}, \beta_{1}^{w}, \beta_{2}^{w}, \beta_{3}^{w}\right) \in(0,1)^{6}$ are independent identically distributed random 6-dimensional vectors indexed by the words $w$ of finite length. Then with probability one harmonic functions are continuous.

(2) Suppose that there is $\varepsilon>0$ such that $\alpha_{j}^{w}, \beta_{j}^{w} \in[\varepsilon, 1-\varepsilon]$ for all $w, j$. Then harmonic functions are Hölder continuous with Hölder exponent $1-\varepsilon^{2}$.

Proof. Under the assumptions of part (1) of the corollary, the $\delta_{w}$ are independent identically distributed random variables which are strictly positive. Therefore the conclusion holds by (5.7) and the Law of Large Numbers.

Part (ii) follows from Theorem 5.5 and the definition of $\delta_{w}$. 


\section{Geometric interpretation}

Algorithm 5.1 can be uniquely presented (up to a constant factor) in geometric terms. This presentation is based on an idea of Kigami to consider a pair of harmonic functions as coordinates for SG [Ki3].

To each point $p \in \mathrm{SG}$ there corresponds a point $\left(h_{3}(p), h_{2}(p)\right) \in \mathbb{R}^{2}$. If harmonic functions separate points - and we always assume this - then the correspondence is one-to-one. In this section we identify $p$ and $\left(h_{3}(p), h_{2}(p)\right)$. This yields a different embedding $\mathrm{SG} \subset \mathbb{R}^{2}$. We have $q_{1}=(0,0), q_{2}=(0,1)$, $q_{3}=(1,0)$. Also, we assume that a harmonic function changes linearly along each resistor, and so a resistor corresponds to a line segment in $\mathbb{R}^{2}$.

For $A, B, C \in \mathbb{R}^{2}$ we denote by $T(A, B, C)$ the triangle with vertices $A, B, C$.

It is easy to see that choosing the matrix $H$ is equivalent to choosing three points $x, y, z$ inside of $T\left(q_{1}, q_{2}, q_{3}\right)$. The positivity of the determinants in Theorem 3.3 is equivalent to the fact that none of the line segments shown in Figure 6.1 intersect or coincide.

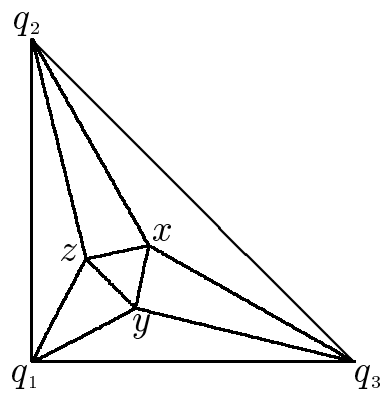

Figure 6.1. Geometric interpretation of conditions of Theorem 3.3.

The Monotonicity Lemma 4.1 corresponds to the fact that the triangles of level $n+1$ are nested inside of those of level $n$ (Figure 6.2).

Going back to Section 5, choosing conductances $R_{1}, R_{2}, R_{3}$ up to a constant factor corresponds to choosing a point $a$ inside of $T\left(q_{1}, q_{2}, q_{3}\right)$. The coordinates of this point $a$ can be thought of as the values of the harmonic functions $h_{3}, h_{2}$ at the imaginary point $a$, the point of intersection of the resistances $R_{1}, R_{2}, R_{3}$. This point is imaginary in the sense that it may not correspond to any point in SG. The point $a$ may be thought of as the center of mass of SG. Thus Step 1 is choosing a point $a$ inside of $T\left(q_{1}, q_{2}, q_{3}\right)$. Step 2 is choosing $a_{j}$ in each of the line segments $\left[q_{j}, a\right], j=1,2,3$, and drawing a new triangle $T\left(a_{1}, a_{2}, a_{3}\right)$ instead of line segments $\left[a_{j}, a\right]$. Step 3 is choosing points $x, y, z$ inside of the line segments $\left[a_{j}, a_{j+1}\right]$.

We summarize the preceding discussion as follows: 


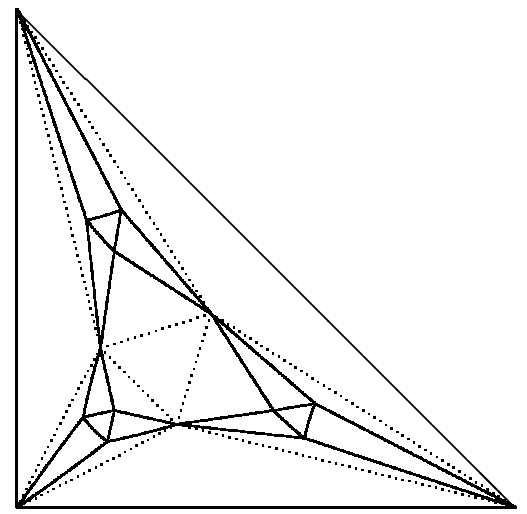

Figure 6.2. Geometric interpretation of the Monotonicity Lemma 4.1 for $n=2$.
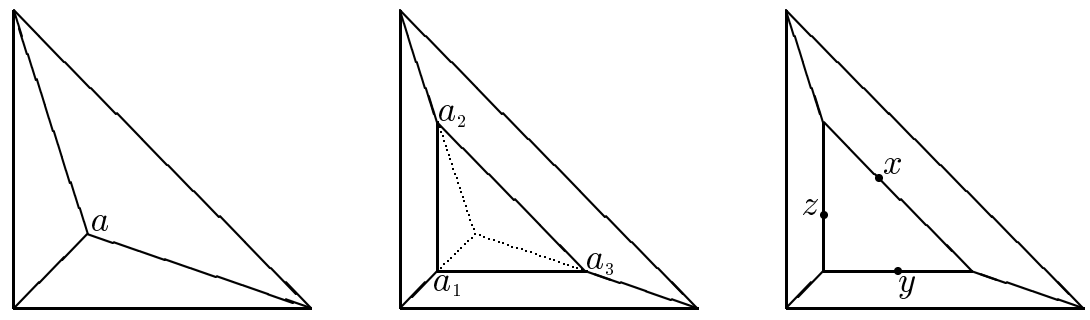

Figure 6.3. Geometric interpretation of the construction in $\S 5$.

Proposition 6.1. Let distinct points $x, y, z \in T\left(q_{1}, q_{2}, q_{3}\right)$ correspond to a matrix $H$ as described above. Then $H$ corresponds to positive conductances $c_{1}, \ldots, c_{9}$ if and only if there are three points $a_{j}$ inside of triangles $T\left(q_{1}, y, z\right), T\left(q_{2}, x, z\right), T\left(q_{3}, x, y\right)$ such that:

(1) $x \in\left[a_{2}, a_{3}\right], y \in\left[a_{1}, a_{3}\right], z \in\left[a_{2}, a_{3}\right]$,

(2) three straight lines from $q_{j}$ to $a_{j}$ intersect in a single point a. 


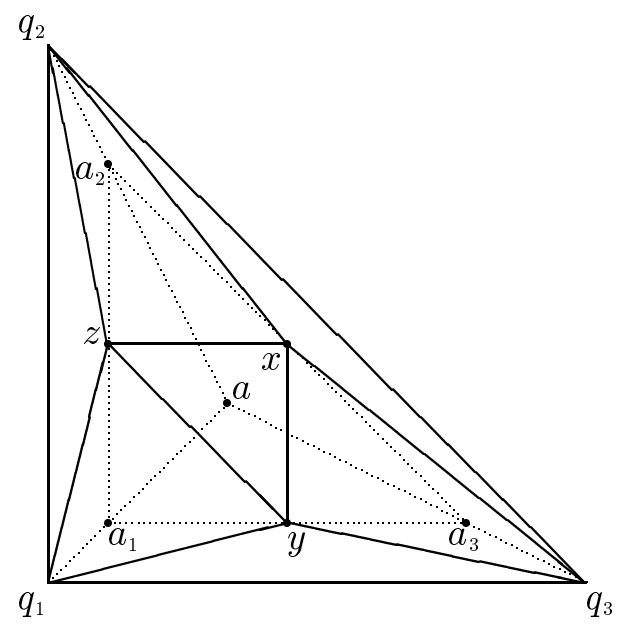

Figure 6.4. Geometric interpretation of Proposition 6.1.

\section{Effective resistance metric, Green's function and capacity of points}

We first recall from [Ki4] some facts about limits of resistance networks. Although we state all the results of this section for the Sierpiński gasket, they can be applied to general pcf fractals with only minor changes.

Let $\mathcal{E}(f, f)$ be defined by (1.2) for any function $f$ on $V_{*}$, where $\mathcal{E}_{n}$ is a compatible sequence of Dirichlet forms on $\Gamma_{n}$.

Proposition 7.1. Every point of $V_{*}=\bigcup_{n \geq 0} V_{n}$ has positive capacity.

Proof. Let $x \in V_{*}$. Then $x \in V_{n}$ for some $n$. The capacity of $\{x\}$ with respect to $\mathcal{E}$ is the same as that with respect to $\mathcal{E}_{n}$ because of the compatibility of the sequence of networks. The latter capacity is positive because $V_{n}$ is a finite set.

The effective resistance is defined for any $x, y \in V_{*}$ by

$$
R(x, y)=\left(\min _{u}\{\mathcal{E}(u, u): u(x)=1, u(y)=0\}\right)^{-1} .
$$

Here the minimum is taken over all functions on $V_{*}$. Note that $x, y \in V_{n}$ for large enough $n$ and that (7.1) does not change if $\mathcal{E}$ is replaced by $\mathcal{E}_{n}$, because of the compatibility condition (see [Ki4], Proposition 2.1.11). By Theorem 2.1.14 in [Ki4], $R(x, y)$ is a metric on $V_{*}$. In what follows we will write $R$-continuity, $R$-closure etc. for continuity, closure etc. with respect to the effective resistance metric $R$. It is known that if $\mathcal{E}(u, u)<\infty$ then $u$ is $R$-continuous ([Ki4], Theorem 2.2.6(1)). The main ingredient in the proof of this fact is the inequality

$$
|u(x)-u(y)|^{2} \leq R(x, y) \mathcal{E}(u, u) .
$$

Let $\Omega$ be the $R$-completion of $V_{*}$. We can conclude from (7.2) that if $u$ is a function on $V_{*}$ such that $\mathcal{E}(u, u)<\infty$ then $u$ has a unique continuation 
to $\Omega$ that is $R$-continuous. We will denote this continuation by the same symbol $u$ and the set of such functions by $\mathcal{F}$.

Remark. It is proved in Proposition 3.3.2 of [Ki4] that if harmonic functions are continuous then there is a continuous injective map $\theta: \Omega \rightarrow \mathrm{SG}$ which is the identity on $V_{*}$. Therefore in this case we can (and will) consider $\Omega$ as a subset of SG. Then $\Omega$ is the $R$-closure of $V_{*}$. In a sense, $\Omega$ is the set where the Dirichlet form lives. If $\Omega$ is not just an abstract completion then the name "Dirichlet form on the SG" is more justified. Strictly speaking, Proposition 3.3.2 in [Ki4] is formulated for self-similar harmonic structures, but self-similarity is not used in the proof.

An important question is whether $\Omega$ is equal to $\mathrm{SG}$. The answer is positive if all the conductances tend to infinity. This happens, for example, in the case of a regular self-similar harmonic structure. Thus it is natural to say that a harmonic structure is regular if $\Omega=\mathrm{SG}$ and nonregular otherwise. It is easy to see that a harmonic structure is regular if all the conductances tend to infinity, but in Example 7.5 we show that the converse is not true. In Example 7.4 we describe a nonregular harmonic structure on SG where harmonic functions are Hölder continuous.

The proof of the next theorem is a part of the proof of Theorem 3.5.6 in [Ki4].

Theorem 7.2. If $x \in \Omega$ then $\{x\}$ has positive capacity.

Proof. Let $\mathcal{F}_{0}=\left\{u \in \mathcal{F}:\left.u\right|_{V_{0}}=0\right\}$. Then $\left(\mathcal{F}_{0}, \mathcal{E}\right)$ is a Hilbert space. If $x \in \Omega, u \in \mathcal{F}_{0}$ and $p \in V_{0}$, then $|u(x)|^{2} \leq R(x, p) \mathcal{E}(u, u)$ and so $u \mapsto u(x)$ is a continuous functional on the Hilbert space $\left(\mathcal{F}_{0}, \mathcal{E}\right)$. Hence there exists $h \in F_{0}$ such that $u(x)=\mathcal{E}(u, h)$ for any $u \in \mathcal{F}_{0}$. This implies the result by $[\mathbf{F u}]$ or $[\mathrm{FOT}]$.

The converse of this statement is proved in [Ki4] for any self-similar harmonic structure. If harmonic functions are continuous then $\Omega$ is just the $R$-closure of $V_{*}$ in SG.

To define the Green's function we use the construction invented by Kigami for self-similar harmonic structures. Let $G=X^{-1}$, where $X=X_{o}+X_{i}$ is defined by (2.1) and (2.2). We assume that the elements $G_{p q}$ of $G$ are indexed by $p, q \in V_{1} \backslash V_{0}$ (this set was denoted by $\{x, y, z\}$ in the previous sections). Let $\psi_{p}$ be the unique piecewise harmonic function such that $\psi_{p}(q)=\delta_{p q}$ for any $p, q \in V_{1}$ and $\psi_{p} \circ F_{j}$ is harmonic for any $j=1,2,3$. We define

$$
\Psi(x, y)=\sum_{p, q \in V_{1} \backslash V_{0}} G_{p q} \psi_{p}(x) \psi_{q}(y) .
$$

For any $p \in V_{1} \backslash V_{0}$ and any $u \in \mathcal{F}_{0}$ we have

$$
\mathcal{E}(\Psi(p, \cdot), u)=u(p)
$$


(see Lemma 3.5 .4 in $[\mathbf{K i} 4]$ ). Let for any $w \in W_{*}$ we define a Dirichlet form $\mathcal{E}_{w}(u, u)$ on SG by

$$
\mathcal{E}_{w}(u, u)=\lim _{n \rightarrow \infty} \sum_{x, y \in V_{n} \cap F_{w}(\mathrm{SG})} c(x, y)\left(u\left(F_{w}^{-1}(x)\right)-u\left(F_{w}^{-1}(y)\right)\right)^{2}
$$

(the sum here is a part of the sum in (1.1)). Then we define $\Psi_{w}(x, y)$ for $\mathcal{E}_{w}(u, u)$ in the same way as $\Psi(x, y)$ is defined for $\mathcal{E}(u, u)$. (This definition differs by a factor of $r_{w}$ from the one in [Ki4].) Finally, Green's function is

$$
g(x, y)=\sum_{w \in W_{*}} \Psi_{w}(x, y) .
$$

Since all the terms in this sum are nonnegative, it either converges or is $+\infty$. Moreover, if $x$ and $y$ are distinct, all but a finite number of terms vanish. Hence if harmonic functions are continuous then $g(x, y)$ is continuous on the set $\{(x, y): x, y \in \mathrm{SG}, x \neq y\}$.

Theorem 7.3. If $x \in \Omega$ then $g(x, x)<\infty, g(x, \cdot) \in \mathcal{F}_{0}$,

$$
\mathcal{E}(g(x, \cdot), u)=u(x)
$$

for any $u \in \mathcal{F}_{0}$, and $\mathcal{E}(g(x, \cdot), h)=0$ for any harmonic function $h$.

Proof. Let

$$
g_{n}(x, y)=\sum_{k=0}^{n} \sum_{w \in W_{k}} \Psi_{w}(x, y) .
$$

Then $g_{n}(x, \cdot) \in \mathcal{F}_{0}$ and for any $u \in \mathcal{F}_{0}$

$$
\mathcal{E}\left(g_{n}(x, \cdot), u\right)=u_{n}(x),
$$

where $u_{n}$ is the unique piecewise harmonic function that coincides with $u$ on $V_{n}$.

It is easy to see that $\mathcal{E}\left(u-u_{n}, u_{n}\right)=0$ and $\mathcal{E}\left(u_{n}, u_{n}\right) \rightarrow \mathcal{E}(u, u)$ as $n \rightarrow 0$. Hence $\mathcal{E}\left(u-u_{n}, u-u_{n}\right) \rightarrow 0$ as $n \rightarrow 0$. Therefore $u_{n}(x) \rightarrow u(x)$ as $n \rightarrow 0$ by (7.2). This implies (7.5) since $g_{n+1}(x, \cdot)-g_{n}(x, \cdot)$ is an $\mathcal{E}$-orthogonal sequence and $u \mapsto u(x)$ is a bounded functional on $\mathcal{F}_{0}$. Therefore $g(x, \cdot)$ lies in $\mathcal{F}_{0}$ and $g(x, x)=\mathcal{E}(g(x, \cdot), g(x, \cdot))<\infty$.

This theorem and its converse are proved in [Ki4] for any self-similar harmonic structure. Again, if harmonic functions are continuous the result holds for points in the $R$-closure of $V_{*}$ in SG.

Example 7.4. It is easy to construct an example of a nonregular self-similar harmonic structure on SG using a modified self-similar structure. Consider the set of 27 contractions $\left\{F_{w}:|w|=3\right\}$, each with contraction ratio $\frac{1}{8}$. We choose constants $r_{w}:|w|=3$ (see [Ki4]) as follows: we choose two numbers $r, r^{\prime}$ and then set $r_{i j k}=r$ if all indices $i, j, k$ are different and $r_{i j k}=r^{\prime}$ otherwise. It is easy to see that, because of symmetry, for any choice of $r>0$ there is an $r^{\prime}>0$ that gives a self-similar harmonic structure. 
(The structure is dihedral-3 symmetric and so has the spectral decimation property, although of a complicated form. See [MT].)

This harmonic structure is nonregular if we choose $r \geq 1$. However it is evident that the parameters described in Section 5 satisfy the condition of Corollary 5.6(2) for any self-similar harmonic structure. Therefore harmonic functions are Hölder continuous.

Example 7.5. We show that conductances need not tend to infinity for a regular harmonic structure. We construct a Dirichlet form $\mathcal{E}$ which is not self-similar, although it is "piecewise self-similar". It is clear from the construction that harmonic functions are continuous and the $R$-topology is equivalent to the standard one.

First, we define an auxiliary family of Dirichlet forms $\mathcal{E}^{\delta}$ as follows: let $\mathcal{E}_{0}^{\delta}$ be the standard symmetric Dirichlet form on $\Gamma_{0}$ for which all three conductances are equal to one. For $\mathcal{E}_{1}^{\delta}$ let the inner conductances be equal to $\delta>0$ and the outer conductances be such that $\mathcal{E}_{1}^{\delta}$ is compatible with $\mathcal{E}_{0}^{\delta}$. Clearly, this is possible for any choice of positive $\delta$. Then we extend $\mathcal{E}_{1}^{\delta}$ to a regular Dirichlet form $\mathcal{E}^{\delta}$ on SG as in Theorem 3.5.

To construct $\mathcal{E}$ we choose an infinite sequence of finite words $\{w(n)\}_{n \geq 1}$ such that $F_{w(n)}(\mathrm{SG}) \bigcap F_{w(m)}(\mathrm{SG})=\varnothing$ if $n \neq m$. Then we choose a sequence of positive numbers $\left\{\delta_{n}\right\}_{n \geq 1}$ such that $\delta_{n} \leq\left(\frac{3}{5}\right)^{|w(n)|}$. Finally, we take the standard Dirichlet form on SG and replace it on each triangle $F_{w(n)}(\mathrm{SG})$ by $\left(\frac{5}{3}\right)^{|w(n)|} \mathcal{E}^{\delta_{n}}\left(f \circ F_{w(n)}^{-1}, f \circ F_{w(n)}^{-1}\right)$.

Note added in proof. In [Ki5], a work submitted after our paper was completed, Kigami proves some extensions of results from his book [Ki4] to a wider context which includes all the Dirichlet forms considered in this paper.

\section{References}

[ASST] B. Adams, S.A. Smith, R. Strichartz and A. Teplyaev, The spectrum of the Laplacian on the pentagasket, Fractals in Graz 2001, Trends Math., Birkhäuser Basel, 2003, 1-24.

[B] M.T. Barlow, Diffusion on Fractals, in 'Lectures on probability theory and statistics' (Saint-Flour, 1995), Lecture Notes Math., 1690, Springer, Berlin, 1998, 1-121, MR 1668115 (2000a:60148), Zbl 0916.60069.

[Fu] M. Fukushima, Dirichlet forms, diffusion processes and spectral dimensions for nested fractals, in 'Ideas and methods in mathematical analysis, stochastics, and applications' (Oslo, 1988), Cambridge Univ. Press, Cambridge, 1992, 151-161, MR 1190496 (94d:60129), Zbl 0764.60081.

[FOT] M. Fukushima, Y. Oshima and M. Takeda, Dirichlet Forms and Symmetric Markov Processes, Studies in Math., 19, de Gruyter, Berlin, 1994, MR 1303354 (96f:60126), Zbl 0838.31001.

[Ki1] J. Kigami, A harmonic calculus on the Sierpiński spaces, Japan J. Appl. Math., 6 (1989), 259-290, MR 1001286 (91g:31005), Zbl 0686.31003. 
[Ki2] J. Kigami Harmonic calculus on p.c.f. self-similar sets, Trans. Amer. Math. Soc., 335 (1993), 721-755, MR 1076617 (93d:39008), Zbl 0773.31009.

[Ki3] J. Kigami Harmonic metric and Dirichlet form on the Sierpiński gasket, in 'Asymptotic problems in probability theory: stochastic models and diffusions on fractals' (K.D. Elworthy and N. Ikeda, eds.), Pitman Research Notes in Math., 283, Longman, 1993, 201-218, MR 1354156 (96m:31014), Zbl 0793.31005.

[Ki4] J. Kigami Analysis on Fractals, Cambridge Tracts in Mathematics, 143, Cambridge University Press, 2001, MR 1840042 (2002c:28015), Zbl 0998.28004.

[Ki5] J. Kigami, Harmonic analysis for resistance forms, J. Funct. Anal., 204 (2003), 399-444, MR 2017320.

$[\mathrm{Ku}]$ S. Kusuoka, Dirichlet forms on fractals and products of random matrices, Publ. Res. Inst. Math. Sci., 25 (1989), 659-680, MR 1025071 (91m:60142), Zbl 0694.60071.

[MT] L. Malozemov and A. Teplyaev, Self-similarity, operators and dynamics, Math. Phys. Anal. Geom., 6 (2003), 201-218, MR 1997913 (2004d:47012), Zbl 1021.05069.

[Me] V. Metz, Self-similar fractals and self-similar energies, Fractals in Graz 2001, Trends Math., Birkhäuser Basel, 2003, 225-240.

[Sa] C. Sabot, Existence and uniqueness of diffusions on finitely ramified self-similar fractals, Ann. Sci. École Norm. Sup (4), 30 (1997), 605-673, MR 1474807 (98h:60118), Zbl 0924.60064.

[SST] J. Stanley, R. Strichartz and A. Teplyaev, Energy partition on fractals, Indiana Univ. Math. J., 52 (2003), 133-156, MR 1970024 (2004a:31006).

[Te] A. Teplyaev, Energy and Laplacian on the Sierpinsski gasket, to appear in Mandelbrot Jubilee, Proc. Sympos. Pure Math., American Mathematical Society, Providence.

Received September 15, 2001. The first author's research was supported by the National Science Foundation through the Research Experiences for Undergraduates Program at Cornell University. The second author's research was supported in part by the National Science Foundation, Grant DMS 9970337. The third author's research was supported by a National Science Foundation Postdoctoral Fellowship.

New College

UNIVERSITY OF SOUTH FLORIDA

TAMPA FL 33620

E-mail address: meyersr@cims.nyu.edu

Courant Institute

NYU

NEW YoRK NY 10012

Mathematics Department

CORnEll University

ITHACA NY 14853

E-mail address: str@math.cornell.edu

Mathematics Department

University of Connecticut

STORRs CT 06269

E-mail address: teplyaev@math.uconn.edu 